\title{
Monotone Difference Approximations for Scalar Conservation Laws
}

\author{
By Michael G. Crandall and Andrew Majda*
}

\begin{abstract}
A complete self-contained treatment of the stability and convergence properties of conservation-form, monotone difference approximations to scalar conservation laws in several space variables is developed. In particular, the authors prove that general monotone difference schemes always converge and that they converge to the physical weak solution satisfying the entropy condition. Rigorous convergence results follow for dimensional splitting algorithms when each step is approximated by a monotone difference scheme.

The results are general enough to include, for instance, Godunov's scheme, the upwind scheme (differenced through stagnation points), and the Lax-Friedrichs scheme together with appropriate multi-dimensional generalizations.
\end{abstract}

Introduction. Perhaps the simplest mathematical models exhibiting behavior typical of that encountered in inviscid continuum mechanics are the initial-value problems for a scalar conservation law. These problems are of the form

$$
\left\{\begin{array}{l}
\text { (i) } u_{t}+\sum_{i=1}^{N} f_{i}(u)_{x_{i}}=0, \text { for } t>0, x=\left(x_{1}, \ldots, x_{N}\right) \in \mathbf{R}^{N}, \\
\text { (ii) } u(x, 0)=u_{0}(x), \text { for } x \in \mathbf{R}^{N},
\end{array}\right.
$$

where the $f_{i}$ are smooth real-valued functions and $u$ is a scalar. It is well known (see [14]) that even if the initial value $u_{0}$ is smooth, the solution to (0.1) typically develops discontinuities as $t$ increases to some $t_{0}>0$ (i.e. shock waves form). Thus the differential equation must be understood in a generalized or weak sense. However, there can be an infinite number of generalized solutions of $(0.1)$ with the same initial data $u_{0}$; and an additional principle, the entropy condition, is needed to select the unique "physical" weak solution (see [14]).

The main new result of this work establishes the convergence of general conservation-form, monotone difference approximations to $(0.1)$ to the unique generalized solution which satisfies the entropy condition. For notational simplicity in the sequel we restrict the presentation to the case $N=2$ for the most part. The corresponding definitions and results for the general case will be clear from this. For $N=2$ we write $(x, y)$ rather than $\left(x_{1}, x_{2}\right)$. Selecting mesh sizes $\Delta x, \Delta y, \Delta t>0$, the value

Received February 12, 1979.

AMS (MOS) subject classifications (1970). Primary 65M10, $65 \mathrm{M05}$.

Key words and phrases. Conservation laws, shock waves difference approximations, entropy conditions.

* Partially supported by Sloan Foundation Fellowship. Sponsored by the United States Army under Contract No. DAAG29-75-C-0024. 
of our numerical approximation at $(j \Delta x, k \Delta y, n \Delta t)$ will be denoted by $U_{j, k}^{n}$. Capital letters $U, V$, etc. will denote functions on the $x, y$ lattice $\Delta=\{(j \Delta x, k \Delta y): j, k$ are integers $\}$; and the value of $U$ at $(j \Delta x, k \Delta y)$ will be written $U_{j, k}$. Thus $U^{n}$, the state of our numerical approximation at the level $n \Delta t$, is a function on $\Delta$ with values $U_{j, k}^{n}$.

The standard notations $\lambda^{x}=\Delta t / \Delta x, \lambda^{y}=\Delta t / \Delta y,\left(\Delta_{+}^{x} U\right)_{j, k}=U_{j+1, k}-U_{j, k}$, $\left(\Delta_{+}^{y} U\right)_{j, k}=U_{j, k+1}-U_{j, k}$, etc., will be used. The difference approximations of $(0.1)$ of interest here are explicit marching schemes of the form

$$
U_{j, k}^{n+1}=G\left(U_{j-p, k-r}^{n}, \ldots, U_{j+q+1, k+s+1}^{n}\right),
$$

where $p, s, q, r$ are nonnegative integers and $G$ is a function of $(p+q+2)(r+s+2)$ real variables. (We are ignoring $\lambda^{x}, \lambda^{y}$ dependence for the moment, as these quantities will typically be fixed.) To simplify notation, $(0.2)$ will be written as

$$
U^{n+1}=\vec{G}\left(U^{n}\right),
$$

with the choice of $p, s, q, r$ dictated by the context. The difference approximation (0.2)-(0.3) is said to have conservation form (see [10]) if there are functions $g_{1}, g_{2}$ such that

$$
\begin{aligned}
\vec{G}(U)_{j, k}= & G\left(U_{j-p, k-r}, \ldots, U_{j+q+1, k+s+1}\right) \\
= & U_{j, k}-\lambda^{x} \Delta_{+}^{x} g_{1}\left(U_{j-p, k-r}, \ldots, U_{j+q, k+s+1}\right) \\
& -\lambda^{y} \Delta_{+}^{y} g_{2}\left(U_{j-p, k-r}, \ldots, U_{j+q+1, k+s}\right) .
\end{aligned}
$$

In order that $(0.3)$ be consistent with $(0.1)$ when $(0.4)$ holds we must have

$$
g_{i}(u, \ldots, u)=f_{i}(u) \text { for } u \in \mathbf{R} \text { and } i=1,2 \text {. }
$$

The functions $g_{i}$ are called the numerical fluxes of the approximation. Finally, the difference approximation is monotone on the interval $[a, b]$ if $G\left(\alpha_{1}, \ldots, \alpha_{(p+q+2)(r+s+2)}\right)$ is a nondecreasing function of each argument $\alpha_{i}$ so long as all arguments lie in $[a, b]$.

It follows from the results of [13] that for $u_{0} \in L^{\infty}\left(\mathbf{R}^{2}\right) \cap L^{1}\left(\mathbf{R}^{2}\right)$ there is a unique weak solution $u \in L^{\infty}\left(\mathbf{R}^{2} \times[0, \infty)\right)$, which satisfies the entropy condition of [13]. (See Section 2 for more details.) Moreover, we can write $u(x, y, t)=\left(S(t) u_{0}\right)$. $(x, y)$, where $S(t): L^{1}\left(\mathbf{R}^{2}\right) \cap L^{\infty}\left(\mathbf{R}^{2}\right) \rightarrow L^{1}\left(\mathbf{R}^{2}\right) \cap L^{\infty}\left(\mathbf{R}^{2}\right)$ for each $t \geqslant 0$ and $t \rightarrow$ $S(t) u_{0}$ is continuous into $L^{1}\left(R^{2}\right)$. To compute this solution numerically we set

$$
U_{j, k}^{0}=\frac{1}{\Delta x \Delta y} \int_{\mathbf{R}_{j, k}} u_{0}(x, y) d x d y,
$$

where

$$
R_{j, k}=[(j-1 / 2) \Delta x,(j+1 / 2) \Delta x) \times[(k-1 / 2) \Delta y,(k+1 / 2) \Delta y),
$$

and define $U^{n+1}$ from $U^{n}$ by (0.3). Finally, put $\chi_{j, k}^{n}=$ characteristic function of $R_{j, k}$ $\times[n \Delta t,(n+1) \Delta t)$ and

$$
u^{\Delta t}=\sum_{n=0}^{\infty} \sum_{j, k=-\infty}^{\infty} U_{j, k}^{n} \chi_{j, k}^{n} .
$$


The main result is

THEOREM 1. Suppose $u_{0} \in L^{1}\left(\mathbf{R}^{2}\right) \cap L^{\infty}\left(\mathbf{R}^{2}\right)$ and $a \leqslant u_{0} \leqslant b$ a.e. Let $(0.2)$ be a consistent conservation-form difference approximation to (0.1)(i) which is monotone on $[a, b]$ and which has Lipschitz continuous numerical fluxes $g_{i}, i=1,2$. Let $u^{\Delta t}$ be given by (0.2), (0.6), (0.7). Then as $\Delta t \rightarrow 0$ with $\lambda^{x}, \lambda^{y}$ fixed, $u^{\Delta t}$ converges to $S(t) u_{0}$ in $L^{1}\left(\mathbf{R}^{2}\right)$ uniformly for bounded $t \geqslant 0$. More precisely,

$$
\lim _{\Delta t \rightarrow 0} \sup _{0 \leqslant t \leqslant T} \iint_{\mathrm{R}^{2}}\left|u^{\Delta t}(x, y, t)-S(t) u_{0}(x, y)\right| d x d y=0
$$

for each $T>0$.

Reviewing the definitions, $(0.8)$ can also be restated as

$$
\lim _{\Delta t \rightarrow 0} \sup _{\substack{0 \leqslant t \leqslant T \\ n \Delta t \leqslant t<(n+1) \Delta t}} \sum_{j, k} \int_{R_{j, k}}\left|U_{j, k}^{n}-S(t) u_{0}(x, y)\right| d x d y=0 .
$$

It should be recalled that even if $N=1$ and $f_{1}$ is convex, nonmonotone schemes such as the Lax-Wendroff scheme can converge to solutions which violate the entropy condition; see [10] and [18]. The result of Theorem 1 applies to the popular dimensional splitting algorithms; see [8], [17], [21]. This follows from simple observations. For example, consider the one-dimensional conservation laws

$$
\left\{\begin{array}{l}
\text { (i) } v_{t}+f_{1}(v)_{x}=0 \\
\text { (ii) } w_{t}+f_{2}(w)_{y}=0 .
\end{array}\right.
$$

If

$$
\left\{\begin{array}{l}
\text { (i) } V_{j}^{n+1}=G_{1}\left(V_{j-p}^{n}, \ldots, V_{j+q+1}^{n}\right), \\
\text { (ii) } W_{k}^{n+1}=G_{2}\left(W_{k-r}^{n}, \ldots, W_{k+s+1}^{n}\right)
\end{array}\right.
$$

are conservation-form difference approximations of (0.10)(i), (ii), respectively, Lax has observed that the scheme defined by

$$
\left\{\begin{array}{l}
U_{j, k}^{n+1}=G_{1}\left(U_{j-p, k}^{n+1 / 2}, \ldots, U_{j+q+1, k}^{n+1 / 2}\right) \\
U_{l, m}^{n+1 / 2}=G_{2}\left(U_{l, m-r}^{n+r}, \ldots, U_{l, m+s+1}^{n}\right)
\end{array}\right.
$$

has conservation form and is consistent with (0.1); see [2] or check the definitions. When $G_{1}$ and $G_{2}$ are also monotone on $[a, b]$ the scheme in (0.12) will be monotone on $[a, b]$. (Since $a \leqslant V_{j+l} \leqslant b$ for $-p \leqslant l \leqslant q+1$ implies $a \leqslant G_{1}\left(V_{j-p}, \ldots, V_{j+q+1}\right.$ ) $\leqslant b$ by Proposition 3.1(b) below, this is evident.) Thus, Theorem 1 applies to the split scheme (0.12). This fact, along with other results concerning splitting algorithms is developed in [17].

The plan of this work is as follows: In Section 1 various monotone difference schemes to which Theorem 1 applies are recalled. These include the Lax-Friedrichs scheme, the upwind scheme (differenced through stagnation points) and Godunov's scheme. The construction of a wide variety of multi-dimensional schemes from the 
above one-dimensional ones is discussed. In view of these examples, many of the results of Le Roux [16] for specific schemes in a single space dimension are included in our general approach. Section 2 is a review of some basic facts about solutions to (0.1) and some function spaces and estimates needed in the proof of Theorem 1 . We discuss the stability of monotone conservation-form difference schemes in Section 3. In particular, we prove that such schemes define $L^{1}$ contractions; this was proved by Jennings [11] in the case of one space variable, but even there our proof, based on a lemma of Crandall and Tartar [5], is simpler. In Section 4 we verify that if solutions of monotone conservation-form difference approximations converge, the limit satisfies the entropy conditions. This was proved by Harten, Hyman and Lax in [10] for $N=$ 1 ; however, we build a different discrete entropy flux which yields a simpler proof for general $N$ and requires only continuity of the numerical fluxes. This generality is useful for applications. (See Section 1 and [16].) The various results of Sections 2, 3, 4 are pieced together to prove Theorem 1 in Section 5. In Section 6 we briefly discuss the inhomogeneous equation.

In fact, our arguments yield more than Theorem 1 states, for the existence of the solution $S(t) u_{0}$ of $(0.1)$ is established while proving convergence (see Section 5). See Conway and Smoller [3], Douglis [6], and Kojima [12] for earlier uses of the (monotone) Lax-Friedrichs difference approximation to prove existence. Oharu and Takahashi prove this scheme converges to the solution satisfying the entropy condition via nonlinear semigroup methods in [19].

Added in Proof. Kuznecov and Vološin [23], a paper we uncovered on the day it was necessary to return the corrected proofs, states the main result of this work together with an error estimate under stronger regularity assumptions than used here.

1. Examples. In this section we present a variety of difference schemes to which Theorem 1 applies. Later sections are independent of the current one.

We begin with several well-known schemes in the case $N=1$. So long as $N=1$ we will write $f, g$ in place of $f_{1}, g_{1}$. For a single-space variable the Lax-Friedrichs scheme is given by

$$
u_{j}^{n+1}=u_{j}^{n}-\frac{\lambda^{x}}{2} \Delta_{0}^{x} f\left(u_{j}^{n}\right)+\frac{1}{2} \Delta_{+}^{x} \Delta_{-}^{x} u_{j}^{n}
$$

Equivalently,

$$
u_{j}^{n+1}=u_{j}^{n}-\lambda^{x} \Delta_{+}^{x} g\left(u_{j}, u_{j-1}\right),
$$

where the numerical flux $g$ is given by

$$
g\left(u_{j}, u_{j-1}\right)=\frac{f\left(u_{j}\right)+f\left(u_{j-1}\right)}{2}-\frac{1}{2 \lambda^{x}}\left(u_{j}-u_{j-1}\right) .
$$

The conservation form and consistency are obvious. A simple analysis reveals that (1.1) is also monotone on $[a, b]$ provided that the C.F.L. condition

$$
\lambda^{x} \max _{a \leqslant u \leqslant b}\left|f^{\prime}(u)\right| \leqslant 1
$$


holds. More generally, if $\gamma$ is nondecreasing, the scheme

$$
u_{j}^{n+1}=u_{j}^{n}-\frac{\lambda^{x}}{2} \Delta_{0}^{x} f\left(u_{j}^{n}\right)+\frac{1}{2} \Delta_{+}^{x} \Delta_{-}^{x} \gamma\left(u_{j}^{n}\right)
$$

has conservation form with the flux.

$$
g\left(u_{j}, u_{j-1}\right)=\frac{f\left(u_{j}\right)+f\left(u_{j-1}\right)}{2}-\frac{1}{2 \lambda^{x}}\left(\gamma\left(u_{j}\right)-\gamma\left(u_{j-1}\right)\right)
$$

and (1.3) is monotone on $[a, b]$ provided that

$$
1-\lambda^{x} \gamma^{\prime}(u) \geqslant 0 \text { and } \gamma^{\prime}(u)-\lambda^{x}\left|f^{\prime}(u)\right| \geqslant 0 \text { for } a \leqslant u \leqslant b .
$$

If $f$ is nondecreasing, the upwind difference scheme is given by

$$
u_{j}^{n+1}=u_{j}^{n}-\lambda^{x}\left(f\left(u_{j}^{n}\right)-f\left(u_{j-1}^{n}\right)\right)=u_{j}^{n}-\lambda^{x} \Delta_{+}^{x} f\left(u_{j-1}^{n}\right),
$$

while if $f$ is nonincreasing it has the form

$$
u_{j}^{n+1}=u_{j}^{n}-\lambda^{x} \Delta_{+}^{x} f\left(u_{j}^{n}\right) .
$$

Next let $\alpha \in \mathbf{R}$, and assume

$$
f^{\prime}(u) \geqslant 0 \text { for } u \geqslant \alpha \text { and } f^{\prime}(u) \leqslant 0 \text { for } u \leqslant \alpha .
$$

Thus, $f^{\prime}(\alpha)=0$ and $\alpha$ is a stagnation point. Without loss of generality, we may also assume that $f(\alpha)=0$ (since changing $f$ by a constant leaves (0.1) invariant). Set

$$
\theta(s)= \begin{cases}1 & \text { if } s \leqslant \alpha, \\ 0 & \text { if } s>\alpha,\end{cases}
$$

and consider the scheme

$$
u_{j}^{n+1}=u_{j}^{n}-\lambda^{x} \Delta_{+}^{x}\left(\theta\left(u_{j}^{n}\right) f\left(u_{j}^{n}\right)+\left(1-\theta\left(u_{j-1}^{n}\right)\right) f\left(u_{j-1}^{n}\right)\right) .
$$

The resulting scheme (for any choice of $\theta$ ) is clearly consistent, has conservation form, and when $\theta \equiv 0$ or $\theta \equiv 1$ reduces to the conventional upwind schemes (1.5), (1.6). In our case ((1.7) holds, $\theta$ given by $(1.8)$ and $f(\alpha)=0)$ it is easy to verify that (1.10) is monotone on $[a, b]$, provided that (1.2) holds.

The last scheme we mention for $N=1$ is Godunov's method. Here we consider only the case in which $f^{\prime \prime}>0$ (in particular, $f$ is strictly convex). Godunov's method is then given by the three-point conservation-form scheme

$$
u_{j}^{n+1}=u_{j}^{n}-\lambda^{x} \Delta_{+}^{x} g\left(u_{j-1}^{n}, u_{j}^{n}\right),
$$

where the numerical flux $g$ is defined by the complicated recipe:

$$
g\left(u_{j-1}, u_{j}\right)= \begin{cases}f\left(u_{j-1}\right) & \text { if } u_{j-1} \geqslant u_{j} \text { and } f\left(u_{j-1}\right) \geqslant f\left(u_{j}\right), \\ f\left(u_{j}\right) & \text { if } u_{j-1} \geqslant u_{j} \text { and } f\left(u_{j-1}\right) \leqslant f\left(u_{j}\right), \\ f\left(u_{j-1}\right) & \text { if } u_{j-1} \leqslant u_{j} \text { and } f^{\prime}\left(u_{j-1}\right) \geqslant 0, \\ f\left(u_{j}\right) & \text { if } u_{j-1} \leqslant u_{j} \text { and } f^{\prime}\left(u_{j}\right) \leqslant 0, \\ f\left(\left(f^{\prime}\right)^{-1}(0)\right) & \text { otherwise. }\end{cases}
$$


We have written down the function $g$ explicitly because these formulas show that for Godunov's method the numerical flux is Lipschitz continuous but not everywhere differentiable. In order to verify that Godunov's method defines a monotone scheme we recall the basic idea of the method. Given bounded discrete data $u_{j}^{n}$ with $a \leqslant u_{j}^{n} \leqslant b$, let

$$
u_{0}=\sum_{j=-\infty} u_{j}^{n} \chi_{j}
$$

where $\chi_{j}$ is the characteristic function of $(j-1 / 2) \Delta x \leqslant x<(j+1 / 2) \Delta x$. Now we let $u$ be the exact solution of

$$
\left\{\begin{array}{l}
u_{t}+f(u)_{x}=0, \\
u(0, x)=u_{0}(x)
\end{array}\right.
$$

which we write as $u(x, t)=S(t) u_{0}(x)$. Since solutions of (1.11) propogate at finite speed at most $c_{0}=\max _{a \leqslant u \leqslant b} \mid f^{\prime}(u) !$ (see Section 2), it follows that $S^{x}(\Delta t) u_{0}(x)$, restricted to $(j-1 / 2) \Delta x \leqslant x \leqslant(j+1 / 2) \Delta x$, depends only on the three values $u_{j-1}^{n}, u_{j}^{n}$, $u_{j+1}^{n}$ provided that, as we now assume, (1.2) holds. Using the form of the exact solution of (1.12) for $0 \leqslant t \leqslant \Delta t$ (see [14]) and the standard flux balance relation, one explicitly computes

$$
u_{j}^{n+1}=\frac{1}{\Delta x} \int_{(j-1 / 2) \Delta x}^{(j+1 / 2) \Delta x} S^{x}(\Delta t) u_{0}(x) d x
$$

and finds (1.10), (1.11). It is well known (see our proof of Theorem 1) that $v_{0} \geqslant u_{0}$ implies $S(t) v_{0} \geqslant S(t) u_{0}$. Hence, $u_{j}^{n+1}$ is a nondecreasing function of $u_{j-1}^{n}, u_{j}^{n}, u_{j+1}^{n}$, which establishes the monotonicity of the scheme.

Multi-dimensional examples of schemes to which Theorem 1 applies can be built from the one-dimensional schemes in a variety of ways. First, there are the methods of dimensional splitting as mentioned in the introduction. To this we add one additional method. Let

$$
\left\{\begin{array}{l}
\text { (i) } V_{j}^{n+1}=G_{1}\left(V_{j-p}^{n}, \ldots, V_{j+q+1}^{n}\right)=V_{j}^{n}-\lambda^{x} \Delta_{+}^{x} g_{1}\left(V_{j-p}^{n}, \ldots, V_{j+q}^{n}\right), \\
\text { (ii) } W_{k}^{n+1}=G_{2}\left(W_{k-r}^{n}, \ldots, W_{k+s+1}^{n}\right)=W_{j}^{n}-\lambda^{y} \Delta_{+}^{y} g_{2}\left(W_{k-r}^{n}, \ldots, W_{k+s}^{n}\right),
\end{array}\right.
$$

denote two conservation-form schemes consistent with

$$
\left\{\begin{array}{l}
\text { (i) } v_{t}+\left(\frac{1}{\alpha} f_{1}(v)\right)_{x}=0 \\
\text { (ii) } w_{t}+\left(\frac{1}{1-\alpha} f_{2}(w)\right)_{y}=0
\end{array}\right.
$$

respectively, where $0<\alpha<1$. Now form the composite scheme

$$
U_{j, k}^{n+1}=\alpha G_{1}\left(U_{j-p, k}^{n}, \ldots, U_{j+q+1, k}^{n}\right)+(1-\alpha) G_{2}\left(U_{j, k-r}^{n}, \ldots, U_{j, k+s+1}^{n}\right) .
$$

Then (1.15) has conservation form and is consistent with $u_{t}+f_{1}(u)_{x}+f_{2}(u)_{y}=0$. Moreover, if (1.13)(i), (ii) are monotone on $[a, b]$ so then is (1.15). Any of the 
schemes discussed above may be used for $G_{1}, G_{2}$. For example, if $\alpha=1 / 2$ and $G_{1}, G_{2}$ are chosen as Lax-Friedrich's scheme (1.1), then (1.15) reads

$$
u_{j, k}^{n+1}=u_{j, k}^{n}-\frac{\lambda^{x}}{2} \Delta_{0}^{x} f_{1}\left(u_{j, k}^{n}\right)-\frac{\lambda^{y}}{2} \Delta_{0}^{y} f_{2}\left(u_{j, k}^{n}\right)+\frac{1}{4}\left(\Delta_{+}^{x} \Delta_{-}^{x}+\Delta_{+}^{y} \Delta_{-}^{y}\right)\left(u_{j, k}^{n}\right),
$$

which is the Lax-Friedrich's scheme in two dimensions. The C.F.L. condition guaranteeing monotonicity on $[a, b]$ is now

$$
\lambda^{x} \max _{a \leqslant u \leqslant b}\left|f_{1}^{\prime}(u)\right|, \lambda^{y} \max _{a \leqslant u \leqslant b}\left|f_{2}^{\prime}(u)\right| \leqslant \frac{1}{2},
$$

which is a factor of 2 more severe than (1.2).

2. Preliminaries. We begin by recalling some of the basic facts concerning solutions of the problem (0.1). A weak solution of the conservation law (0.1)(i) on $\mathbf{R}^{N} \times$ $[0, T]$ is a function $u \in L^{\infty}\left(\mathbf{R}^{N} \times[0, T]\right)$ such that

$$
\int_{0}^{T} \int_{\mathbf{R}^{N}}\left(\varphi_{t} u+\sum_{i=1}^{N} \varphi_{x_{i}} f_{i}(u)\right) d x d t=0 \quad \text { for every } \varphi \in C_{0}^{1}\left(\mathbf{R}^{N} \times(0, T)\right)
$$

where $C_{0}^{1}\left(\mathbf{R}^{N} \times(0, T)\right)$ denotes the continuously differentiable functions on $\mathbf{R}^{N} \times$ $(0, T)$ with compact support. As we remarked earlier, weak solutions are not uniquely determined by their initial data (properly interpreted); and an additional condition, the entropy condition, is needed to select the desired solution. The form of the entropy condition we will use was given by Vol'pert [22]. An entropy solution of the conservation law (0.1)(i) on $\mathbf{R}^{N} \times(0, T)$ is a function $u \in L^{\infty}\left(\mathbf{R}^{N} \times[0, T]\right)$ such that

$$
\left\{\begin{array}{l}
\int_{0}^{T} \int_{\mathbf{R}^{N}} \varphi_{t}|u-c|+\sum_{i=1}^{N} \varphi_{x_{i}} \operatorname{sgn}(u-c)\left(f_{i}(u)-f_{i}(c)\right) d x d t \geqslant 0 \\
\text { for every } \varphi \in C_{0}^{1}\left(\mathbf{R}^{2} \times(0, T)\right) \text { with } \varphi \geqslant 0 \text { and every } c \in \mathbf{R} .
\end{array}\right.
$$

In (2.2), sgn $r=r /|r|$ for $r \neq 0$ (and the value assigned to sgn 0 is irrelevant since $f_{i}(u)-f_{i}(c)=0$ if $u=c$ ). If $a \leqslant u \leqslant b$ a.e., then choosing $c=b$ and $c=a$ in (2.2) we can deduce (2.1) for $\varphi \geqslant 0$ (and hence, in general). That is, entropy solutions are weak solutions. Moreover, if $a \leqslant u \leqslant b$ a.e. and (2.2) holds for $a \leqslant c \leqslant b$, then it clearly holds for all $c$. The existence and uniqueness of entropy solutions of (0.1) which are of (locally) bounded variation (see below), when $u_{0}$ is of (locally) bounded variation and the initial condition is properly interpreted, was proved by Vol'pert [22] . Subsequently, Kružkov [13] extended these results. A special case of Kružkov's uniqueness theorem adequate for our purposes is:

THEOREM 2.1 (UNIQUeness). Let $u_{10}, u_{20} \in L^{\infty}\left(\mathbf{R}^{N}\right)$. Let $u_{1}, u_{2}$ be entropy solutions of (0.1)(i) on $\mathbf{R}^{N} \times(0, T)$ for which

$$
\underset{t \downarrow 0}{\text { essential limit }} \int_{|x|<R}\left|u_{i}(x, t)-u_{i 0}(x)\right| d x=0 \quad \text { for } R>0, i=1,2 .
$$

Let $L$ be a Lipschitz constant for the mapping $r \rightarrow\left(f_{1}(r), \ldots, f_{N}(r)\right)$ on $|r| \leqslant$ $\max \left\{\left\|u_{i}\right\|_{L}^{\infty}\left(\mathrm{R}^{N} \times(0, T)\right): i=1,2\right\}$. Then 


$$
\left\{\begin{array}{l}
\int_{|x| \leqslant R}\left|u_{1}(x, t)-u_{2}(x, t)\right| d x \leqslant \int_{|x| \leqslant R+L t}\left|u_{10}(x)-u_{20}(x)\right| d x \\
\text { for } R>0 \text { and almost all } t \in[0, T] .
\end{array}\right.
$$

In particular, if $u_{10}=u_{20}$, then $u_{1}=u_{2}$ a.e. If we choose $u_{20}=u_{2}=0$ in (2.4) (constants are entropy solutions of $(0.1)(i)$ ), we find

$$
\int_{|x| \leqslant R}\left|u_{1}(x, t)\right| d x \leqslant \int_{|x| \leqslant R+L t}\left|u_{10}(x)\right| d x .
$$

If $u_{10} \in L^{1}\left(\mathbf{R}^{N}\right)$, letting $R \rightarrow \infty$ above yields

$$
\int_{\mathrm{R}^{N}}\left|u_{1}(x, t)\right| d x \leqslant \int_{\mathrm{R}^{N}}\left|u_{10}(x)\right| d x,
$$

so $t \rightarrow u_{1}(\cdot, t)$ is bounded into $L^{1}\left(\mathbf{R}^{N}\right)$ (or $u_{1} \in L^{\infty}\left(0, T: L^{1}\left(\mathbf{R}^{N}\right)\right)$ ). It will prove convenient for us to work with this case, that is, $u_{0} \in L^{1}\left(\mathbf{R}^{N}\right) \cap L^{\infty}\left(\mathbf{R}^{N}\right)$. It is a simple matter to pass then to the more general case $u_{0} \in L^{\infty}\left(\mathbf{R}^{N}\right)$ via the finite domain of dependence established in (2.4) (or the finite numerical domain of dependence for our schemes).

It follows from the results of [13], [22] that for every $u_{0} \in L^{\infty}\left(\mathbf{R}^{N}\right)(0.1)(\mathrm{i})$ has an entropy solution $u$ assuming the initial-value $u_{0}$ in the sense (2.3). This existence result will follow easily from our investigations, so we will not belabor it here.

Next, we define some function spaces needed in the sequel. From now on we set $N=2$ in this section and assume the reader can extrapolate to the case of general N. $L_{\text {loc }}^{1}\left(\mathbf{R}^{2}\right)$ is the space of functions which are integrable on compact subsets of $\mathbf{R}^{\mathbf{2}}$. A sequence $\left\{g_{n}\right\} \leqslant L_{\text {loc }}^{1}\left(\mathbf{R}^{2}\right)$ converges to $g$ in $L_{\text {loc }}^{1}\left(\mathbf{R}^{2}\right)$ provided $\left\|g_{n}-g\right\|_{L^{1}(K)} \rightarrow$ 0 for every compact $K \subset \mathbf{R}^{2} . B V\left(\mathbf{R}^{2}\right)$ denotes the subspace of $f \in L_{\text {loc }}^{1}\left(\mathbf{R}^{2}\right)$ for which $\|f\|_{B V\left(\mathrm{R}^{2}\right)}<\infty$, where

$$
\begin{aligned}
\|f\|_{B V\left(\mathbf{R}^{2}\right)}= & \sup _{h \in \mathbf{R} \backslash\{0\}} \int_{\mathbf{R}^{2}} \frac{|f(x+h, y)-f(x, y)|}{|h|} d x d y \\
& +\sup _{h \in \mathbf{R} \backslash\{0\}} \int_{\mathbf{R}^{2}} \frac{|f(x, y+h)-f(x, y)|}{|h|} d x d y .
\end{aligned}
$$

We observe that for $f \in B V\left(\mathbf{R}^{2}\right)$ and $h, s \in \mathbf{R}$

$$
\begin{aligned}
& \int_{\mathbf{R}^{2}} \mid f(x+h, y+s)-f(x, y) \mid d x d y \\
& \leqslant \int_{\mathbf{R}^{2}}|f(x+h, y+s)-f(x, y+s)| d x d y \\
&+\int_{\mathbf{R}^{2}}|f(x, y+s)-f(x, y)| d x d y \\
& \leqslant(|h|+|s|)\|f\|_{B V\left(\mathbf{R}^{2}\right)}
\end{aligned}
$$

The space $L^{1}\left(\mathbf{R}^{2}\right) \cap B V\left(\mathbf{R}^{2}\right)$ is equipped with the norm

$$
\|f\|_{L^{1}\left(\mathrm{R}^{2}\right) \cap B V\left(\mathrm{R}^{2}\right)}=\|f\|_{L^{1}\left(\mathrm{R}^{2}\right)}+\|f\|_{B V\left(\mathrm{R}^{2}\right)} .
$$


We will use the following compactness criterion (which is stated with unnecessarily restrictive hypotheses).

Proposition 2.2. Let $H \subset L^{1}\left(\mathbf{R}^{2}\right)$ be bounded and

$$
\lim _{(h, s) \rightarrow(0,0)} \sup _{f \in H} \int_{\mathbf{R}^{2}}|f(x+h, y+s)-f(x, y)| d x d y=0 .
$$

Then $H$ is precompact in $L_{10 c}^{1}\left(R^{2}\right)$.

This is immediate from standard results, e.g., [6, IV.8.21]. As a corollary, we note that (2.6), (2.7) imply

(2.9) bounded subsets of $L^{1}\left(\mathbf{R}^{2}\right) \cap B V\left(\mathbf{R}^{2}\right)$ are precompact in $L_{\text {loc }}^{1}\left(\mathbf{R}^{2}\right)$.

Finally, we summarize several facts which will be used in passing between continuous and discrete estimates. Let us recall the notations. $U, V$, etc. denote functions on the lattice $\Delta=\left\{(j \Delta x, k \Delta y)=\Delta t\left(j / \lambda^{x}, b / \lambda^{y}\right): j, k\right.$ integers $\}$ with values $U_{j, k}$, $V_{j, k}$, etc. We set

$$
\|U\|_{L^{1}(\Delta)}=\sum_{j, k}\left|U_{j, k}\right| \Delta x \Delta y, \quad\|U\|_{L^{\infty}(\Delta)}=\sup _{j, k}\left|U_{j, k}\right|
$$

and

$$
\|U\|_{B V(\Delta)}=\frac{1}{\Delta x}\left\|\Delta_{+}^{x} U\right\|_{L^{1}(\Delta)}+\frac{1}{\Delta y}\left\|\Delta_{+}^{y} U\right\|_{L^{1}(\Delta)},
$$

where $\left(\Delta_{+}^{x} U\right)_{j, k}=U_{j+1, k}-U_{j, k}$, etc. $L^{1}(\Delta), B V(\Delta)$ consist of those $U$ for which (2.10), (2.11) are finite.

The characteristic function of the rectangle $R_{j, k}=[(j-1 / 2) \Delta x,(j+1 / 2) \Delta x) \times$ $[(k-1 / 2) \Delta y,(k+1 / 2) \Delta y)$ is denoted by $\chi_{j, k}$. Given a lattice function $U$ we define a piecewise constant function $U_{\mathbf{R}^{2}}$ on $\mathbf{R}^{2}$ by $U_{\mathbf{R}^{2}}=U_{j, k}$ on $R_{j, k}$. Equivalently,

$$
U_{\mathrm{R}^{2}}=\sum_{j, k} U_{j, k} \chi_{j, k}
$$

Given a function $u \in L_{\text {loc }}^{1}\left(\mathbf{R}^{2}\right)$ we define a lattice function $u_{\Delta}$ by

$$
\left(u_{\Delta}\right)_{j, k}=\frac{1}{\Delta x \Delta y} \int_{R_{j, k}} u(x, y) d x d y
$$

One has

$$
\begin{gathered}
\left\|U_{\mathbf{R}^{2}}\right\|_{L^{1}\left(\mathrm{R}^{2}\right)}=\|U\|_{L^{1}(\Delta)}, \quad\left\|U_{\mathrm{R}^{2}\left\|_{L^{\infty}\left(\mathrm{R}^{2}\right)}=\right\| U \|_{L^{\infty}(\Delta)},}, \quad\right\|\|u\|_{L^{1}\left(\mathrm{R}^{2}\right)}, \quad\left\|u_{\Delta}\right\|_{L^{\infty}(\Delta)} \leqslant\|u\|_{L^{\infty}\left(\mathrm{R}^{2}\right)}, \\
\| u_{L^{1}(\Delta)} \leqslant U_{\mathbf{R}^{2}\left\|_{B V\left(\mathrm{R}^{2}\right)} \leqslant\right\| U \|_{B V(\Delta)},}
\end{gathered}
$$

and

$$
\left\|u_{\Delta}\right\|_{B V(\Delta)} \leqslant\|u\|_{B V\left(\mathrm{R}^{2}\right)}
$$


Of these relations, (2.14) and (2.15) are immediate from the definitions. Also, (2.17) is easy to see for, by the definitions,

$$
\begin{aligned}
\left\|u_{\Delta}\right\|_{B V(\Delta)}= & \sum_{j, k}\left(\frac{1}{\Delta x}\left|\int_{\mathbf{R}_{j+1, k}} u(x, y) d x d y-\int_{R_{j, k}} u(x, y) d x d y\right|\right. \\
& \left.\quad+\frac{1}{\Delta y}\left|\int_{R_{j, k+1}} u(x, y) d x d y-\int_{\mathbf{R}_{j, k}} u(x, y) d x d y\right|\right) \\
= & \sum_{j, k}\left(\frac{1}{\Delta x}\left|\int_{R_{j, k}}(u(x+\Delta x, y)-u(x, y)) d x d y\right|\right. \\
& \left.+\frac{1}{\Delta y}\left|\int_{R_{j, k}} u(x, y+\Delta y)-u(x, y) d x d y\right|\right) \\
\leqslant & \|u\|_{B V\left(\mathbf{R}^{2}\right)}
\end{aligned}
$$

It remains to check (2.17). To this end, observe that

$$
\begin{aligned}
\int_{\mathbf{R}^{2}} \mid f(x+ & \left.h_{1}+h_{2}, y\right)-f(x, y) \mid d x d y \\
\leqslant & \int_{\mathbf{R}^{2}}\left|f\left(x+h_{1}+h_{2}, y\right)-f\left(x+h_{2}, y\right)\right| d x d y \\
& +\int_{\mathbf{R}^{2}}\left|f\left(x+h_{2}, y\right)-f(x, y)\right| d x d y \\
= & \int_{\mathbf{R}^{2}}\left|f\left(x+h_{1}, y\right)-f(x, y)\right| d x d y \\
& +\int_{\mathbf{R}^{2}}\left|f\left(x+h_{2}, y\right)-f(x, y)\right| d x d y .
\end{aligned}
$$

That is, $g\left(h_{1}+h_{2}\right) \leqslant g\left(h_{1}\right)+g\left(h_{2}\right)$, where $g\left(h_{1}+h_{2}\right)$ is the left-hand side of (2.18). Assume now that $h>0$, and write $h=(l \Delta x+\xi \Delta x)$ where $l$ is a nonnegative integer and $0 \leqslant \xi<1$. By the above

$$
\begin{aligned}
\int_{\mathbf{R}^{2}}\left|U_{\mathbf{R}^{2}}(x+h, y)-U_{\mathbf{R}^{2}}(x, y)\right| d x d y \\
\leqslant l \int_{\mathbf{R}^{2}}\left|U_{\mathbf{R}^{2}}(x+\Delta x, y)-U_{\mathbf{R}^{2}}(x, y)\right| d x d y \\
\quad+\int_{\mathbf{R}^{2}}\left|U_{\mathbf{R}^{2}}(x+\xi \Delta x, y)-U_{\mathbf{R}^{2}}(x, y)\right| d x d y
\end{aligned}
$$

By the definitions

$$
\begin{aligned}
\int_{\mathrm{R}^{2}} \mid U_{\mathrm{R}^{2}}(x & +\Delta x, y)-U_{\mathrm{R}^{2}}(x, y) \mid d x d y \\
& =\sum_{j, k}\left|U_{j+1, k}-U_{j, k}\right| \Delta x \Delta y=\left\|\Delta_{+}^{x} U\right\|_{L^{1}(\Delta)}
\end{aligned}
$$


and

$$
\begin{aligned}
\int_{\mathbf{R}^{2}}\left|U_{\mathbf{R}^{2}}(x+\xi \Delta x, y)-U_{\mathbf{R}^{2}}(x, y)\right| d x d y \\
\quad=\sum_{j, k} \int_{R_{j, k}}\left|U_{\mathbf{R}^{2}}(x+\xi \Delta x, y)-U_{j, k}\right| d x d y \\
\quad=\sum_{j, k} \int_{(j+1 / 2-\xi) \Delta x}^{(j+1 / 2) \Delta x} \int_{(k-1 / 2) \Delta y}^{(k+1 / 2) \Delta y}\left|U_{j+1, k}-U_{j, k}\right| d x d y \\
=\sum_{j, k} \xi\left|U_{j+1, k}-U_{j, k}\right| \Delta x \Delta y .
\end{aligned}
$$

Combining (2.19), (2.20), (2.21) and recalling $h=l \Delta x+\xi \Delta x$, we find

$$
\frac{1}{h} \int_{\mathbf{R}^{2}}\left|U_{\mathbf{R}^{2}}(x+h, y)-U_{\mathbf{R}^{2}}(x, y)\right| d x d y \leqslant \frac{1}{\Delta x}\left\|\Delta_{+}^{x} U\right\|_{L^{1}(\Delta)} .
$$

Treating the $y$-variation in a similar way gives (2.16).

3. Stability Properties of Monotone Conservation-Form Schemes. Recall that our schemes have the form

$$
U_{j, k}^{n+1}=G\left(U_{j-p, k-r}^{n}, \ldots, U_{j+q+1, k+s+1}^{n}\right)
$$

which we write as $U^{n+1}=\vec{G}\left(U^{n}\right)$. The first result is

Proposition 3.1. Let (3.1) be a conservation-form difference scheme which is monotone on the interval $[a, b]$. Let $U, V$ satisfy $a \leqslant U_{j, k}, V_{j, k} \leqslant b$ for all $j, k$. Then

$$
U \leqslant V\left(\text { i.e. } U_{j, k} \leqslant V_{j, k} \text { for all } j, k\right) \text { implies } \vec{G}(U) \leqslant \vec{G}(V)
$$

$$
\min _{\substack{j-p \leqslant l \leqslant j+q+1 \\ k-r \leqslant m \leqslant k+s+1}} U_{l, m} \leqslant \vec{G}(U)_{j, k} \leqslant \max _{\substack{j-p \leqslant l \leqslant j+q+1 \\ k-r \leqslant m \leqslant k+s+1}} U_{l, m} \text {. for all } j, k .
$$

$$
\begin{aligned}
\|\vec{G}(U)-\vec{G}(V)\|_{L^{1}(\Delta)} & \leqslant\|U-V\|_{L^{1}(\Delta)} \quad \text { if } U, V \in L^{1} \\
\|\vec{G}(U)\|_{B V(\Delta)} & \leqslant\|U\|_{B V(\Delta)} \quad \text { if } U \in L^{1}(\Delta) .
\end{aligned}
$$

Proof. We will show first that (c) $\Rightarrow$ (d). Indeed, if $\left(\tau_{\Delta x} W\right)_{j, k}=W_{j+1, k}$, we have $\tau_{\Delta x} \vec{G}(U)=\vec{G}\left(\tau_{\Delta x} U\right)$ (that is, $\vec{G}$ computes with grid translations). Then, by (c),

$$
\begin{aligned}
\left\|\Delta_{+}^{x} \vec{G}(U)\right\|_{L^{1}(\Delta)} & =\left\|\tau_{\Delta x} \vec{G}(U)-\vec{G}(U)\right\|_{L^{1}(\Delta)} \\
& =\left\|\vec{G}\left(\tau_{\Delta x} U\right)-\vec{G}(U)\right\|_{L^{1}(\Delta)} \leqslant\left\|\tau_{\Delta x} U-U\right\|_{L^{1}(\Delta)}=\left\|\Delta_{+}^{x} U\right\|_{L^{1}(\Delta)}
\end{aligned}
$$


Similarly, $\left\|\Delta_{+}^{y} \vec{G}(U)\right\|_{L^{1}(\Delta)} \leqslant\left\|\Delta_{+}^{Y} U\right\|_{L^{1}(\Delta)}$. Now (d) follows from the definition (2.11). A moment's reflection shows that (a) is merely a restatement of the assumption that (3.1) is monotone on $[a, b]$. We next verify (b). Letting $V_{j, k}=\sup _{l, m} U_{l, m}=$ $c$ for all $j, k$, we have $G(V)=V(G$ maps constants to themselves by the conservation form). Now $V \geqslant U$ so $c=\vec{G}(V)_{j, k} \geqslant \vec{G}(U)_{j, k}$ for all $j, k$. Estimating below in a similar way yields $\inf _{l, m} U_{l, m} \leqslant U_{j, k} \leqslant \sup _{l, m} U_{l, m}$. The refinement (b) of this result follows from the fact that $G(U)_{j, k}$ depends only on $U_{l, m}$ for $l, m$ in the range indicated in (b).

It remains to prove (c). The following elementary lemma of M. Crandall and L. Tartar is at the heart of the matter. We use the notations $f \vee g=\max (f, g)$ and $f^{+}=f \vee 0$.

LEMMA 3.2. Let $\Omega$ be a measure space and $C \subset L^{1}(\Omega)$ have the property that $f \vee g \in C$ whenever $f, g \in C$. Let $T: C \rightarrow L^{1}(\Omega)$ satisfy

$$
\int_{\Omega} T(f)=\int_{\Omega} f \quad \text { for } f \in C
$$

Then the following three properties of $T$ are equivalent:

$$
f, g \in C \text { and } f \leqslant g \text { a.e. implies } T(f) \leqslant T(g) \text { a.e., }
$$

$$
\begin{gathered}
\int_{\Omega}(T(f)-T(g))^{+} \leqslant \int_{\Omega}(f-g)^{+} \quad \text { for } f, g \in C, \\
\int_{\Omega}|T(f)-T(g)| \leqslant \int_{\Omega}|f-g| \quad \text { for } f, g \in C .
\end{gathered}
$$

The proof of Lemma 3.2 is given at the end of this section. We apply it here with $\Omega=\Delta$ equipped with the discrete measure assigning mass $\Delta x \Delta y$ to each point. Set

$$
C=\left\{U \in L^{1}(\Delta): a \leqslant U_{j, k} \leqslant b \text { for all } j, k\right\},
$$

and $T(U)=\vec{G}(U)$. It follows from Proposition 3.1(b) that

$$
\|\vec{G}(U)\|_{L^{1}(\Delta)} \leqslant(p+q+2)(s+r+2)\|U\|_{L^{1}(\Delta)},
$$

so $\vec{G}: C \rightarrow L^{1}(\Delta)$. For $U \in C$ we have

$$
\int_{\Delta} \vec{G}(U)=\sum_{j, k} G(U)_{j, k} \Delta x \Delta y=\sum_{j, k} U_{j, k} \Delta x \Delta y=\int_{\Delta} U,
$$

because of the conservation form of $G$. Thus, (3.2) is verified in this case. Moreover, Lemma 3.2(a) is the same as Proposition 3.1(a) in this application. Thus, Proposition 3.1(c) holds by the equivalence of Lemma 3.2(a) and (c).

Remark 3.3. In fact, Proposition 3.1(c) and (d) hold even if $U, V \notin L^{1}(\Delta)$, as can easily be shown. 
Next we examine the continuity properties in time of the function constructed as follows: Let $U^{0} \in L^{1}(\Delta)$ be given with $a \leqslant U_{j, k}^{0} \leqslant b$. Set

$$
U^{n+1}=\vec{G}\left(U^{n}\right)=\vec{G}^{n}\left(U^{0}\right) \text { and } u^{\Delta t}=\sum_{n=0}^{\infty} U_{\mathrm{R}^{2}}^{n} \chi^{n}=\sum_{n=0}^{\infty} \sum_{j, k} U_{j, k}^{n} \chi_{j, k}^{n},
$$

where $\chi^{n}$ is the characteristic function of $[n \Delta t,(n+1) \Delta t)$ and $\chi_{j, k}^{n}$ is the characteristic function of $R_{j, k} \times[n \Delta t,(n+1) \Delta t)$.

Proposition 3.4. Let the assumptions of Proposition 3.1 hold. Let $u^{\Delta t}$ be given by (3.3) and $0 \leqslant t_{1} \leqslant t_{2}$. Then

$$
\begin{aligned}
\int_{\mathbf{R}^{2}}\left|u^{\Delta t}\left(x, y, t_{2}\right)-u^{\Delta t}\left(x, y, t_{1}\right)\right| d x d y \\
\leqslant\left(\frac{\left|t_{2}-t_{1}\right|}{\Delta t}+1\right)\left\|\vec{G}\left(U^{0}\right)-U^{0}\right\|_{L^{1}(\Delta)}
\end{aligned}
$$

Proof. Let $n \Delta t \leqslant t_{2}<(n+1) \Delta t, m \Delta t \leqslant t_{1}<(m+1) \Delta t$ so that $u\left(\cdot, \cdot, t_{2}\right)$ $=U_{\mathbf{R}^{2}}^{n}, u\left(\cdot, \cdot t_{1}\right)=U_{\mathbf{R}^{2}}^{m}$. We will show that

$$
\left\|U_{\mathbf{R}^{2}}^{n}-U_{\mathbf{R}^{2}}^{m}\right\|_{L^{1}\left(\mathrm{R}^{2}\right)} \leqslant(n-m)\left\|\vec{G}\left(U^{0}\right)-U^{0}\right\|_{L^{1}(\Delta)},
$$

from which (3.12) follows since $(n-m) \Delta t \leqslant t_{2}-t_{1}+\Delta t$ by the choice of $n$ and $m$. Using Proposition 3.1(b), $a \leqslant U_{j, k}^{n} \leqslant b$ for $n=0,1, \ldots$ and all $j, k$. Then (2.14) and Proposition 3.1(c) yield

$$
\begin{aligned}
\| U_{\mathrm{R}^{2}}^{n}-U_{\mathrm{R}^{2}}^{m} & \left\|_{L^{1}\left(\mathrm{R}^{2}\right)}=\right\| \vec{G}^{n}\left(U^{0}\right)-\vec{G}^{m}\left(U^{0}\right) \|_{L^{1}(\Delta)} \\
& =\left\|\sum_{i=0}^{n-m-1}\left(\vec{G}^{n-i}\left(U^{0}\right)-\vec{G}^{n-(i+1)}\left(U^{0}\right)\right)\right\|_{L^{1}(\Delta)} \\
& \leqslant \sum_{i=0}^{n-m-1}\left\|\vec{G}^{n-i}\left(U^{0}\right)-\vec{G}^{n-(i+1)}\left(U^{0}\right)\right\|_{L^{1}(\Delta)} \\
& \leqslant \sum_{i=0}^{n-m-1}\left\|\vec{G}\left(U^{0}\right)-U^{0}\right\|_{L^{1}(\Delta)}=(n-m)\left\|\vec{G}\left(U^{0}\right)-U^{0}\right\|_{L^{1}(\Delta)},
\end{aligned}
$$

which gives the desired result.

We next need to estimate the quantity $\left\|\vec{G}\left(U^{0}\right)-U^{0}\right\|_{L}^{1}(\Delta)$.

Proposition 3.5. Let $G$ be given by (0.4) where the fluxes $g_{1}, g_{2}$ are Lipschitz continuous. Then

$$
\frac{1}{\Delta t}\|\vec{G}(U)-U\|_{L^{1}(\Delta)} \leqslant L(p+q+2)(r+s+2)\|U\|_{B V(\Delta)},
$$

where $L$ is a Lipschitz constant for $g_{1}, g_{2}$. 
Proof. By (0.4)

$$
\begin{aligned}
& \|\vec{G}(U)-U\|_{L^{1}(\Delta)} \leqslant \sum_{j, k}\left(\lambda^{x} \mid g_{1}\left(U_{j-p+1, k-r}, \ldots, U_{j+q+1, k+s+1}\right)\right. \\
& -g_{1}\left(U_{j-p, k-r}, \ldots, U_{j+q, k+s+1}\right) \\
& +\lambda^{y} \mid g_{2}\left(U_{j-p, k-r+1}, \ldots, U_{j+q+1, k+s+1}\right) \\
& \left.-g_{2}\left(U_{j-p, k-r}, \ldots, U_{j+q+1, k+s}\right) \mid\right) \Delta x \Delta y \\
& \leqslant \sum_{j, k}\left(\frac{\Delta t}{\Delta x} L \sum_{l=j-p}^{j+q} \sum_{m=k-r}^{k+s+1}\left|U_{l+1, m}-U_{l, m}\right|\right. \\
& \left.+\frac{\Delta t}{\Delta y} L \sum_{l=j-p}^{j+q+1} \sum_{m=k-r}^{k+s}\left|U_{l, m+1}-U_{l, m}\right|\right) \Delta x \Delta y \\
& \leqslant(\Delta t) L(p+q+2)(r+s+2)\left(\frac{1}{\Delta x}\left\|\Delta_{+}^{x} U\right\|_{L^{1}(\Delta)}+\frac{1}{\Delta y}\left\|\Delta_{+}^{y} U\right\|_{L^{1}(\Delta)}\right) \\
& =(\Delta t) L(p+q+2)(r+s+2)\|U\|_{B V(\Delta)},
\end{aligned}
$$

whence the result.

The following corollary of these estimates will be what is eventually used.

Corollary 3.6. Let the assumptions of Propositions 3.4 and 3.5 hold. Let $u_{0} \in L^{1}\left(\mathbf{R}^{2}\right), a \leqslant u_{0} \leqslant b$ a.e., and $u^{\Delta t}$ be given by (3.3), where $U^{0}=u_{0 \Delta}$; see (2.13). Then there is a constant $C$ independent of $u_{0}, \Delta t$ such that for $0 \leqslant t_{1}, t_{2}$

$$
\int_{\mathbf{R}^{2}}\left|u^{\Delta t}\left(x, y, t_{2}\right)-u^{\Delta t}\left(x, y, t_{1}\right)\right| d x d y \leqslant C\left(\left|t_{1}-t_{2}\right|+\Delta t\right)\left\|u_{0}\right\|_{B V\left(\mathbf{R}^{2}\right)^{\circ}}
$$

Proof. This is immediate from Propositions 3.4, 3.5 and (2.17).

Proof of Lemma 3.2. First, we prove that (a) implies (b). Let $f, g \in C$. Then $f \vee g=g+(f-g)^{+} \in C$ by assumption, and $T(f \vee g)-T(g) \geqslant 0$ since $T$ is order preserving. Also $T(f)-T(g) \leqslant T(f \vee g)-T(g)$, and so $(T(f)-T(g))^{+} \leqslant T(f \vee g)-$ $T(g)$. Thus

$$
\int_{\Omega}(T(f)-T(g))^{+} \leqslant \int_{\Omega}(T(f \vee g)-T(g))=\int_{\Omega}(f \vee g-g)=\int_{\Omega}(f-g)^{+},
$$

and (b) is established. That (b) implies (c) is trivial. Indeed, if (b) holds,

$$
\begin{aligned}
\int_{\Omega}|T(f)-T(g)| & =\int_{\Omega}(T(f)-T(g))^{+}+\int_{\Omega}(T(g)-T(f))^{+} \\
& \leqslant \int_{\Omega}(f-g)^{+}+\int_{\Omega}(g-f)^{+}=\int_{\Omega}|f-g|
\end{aligned}
$$

Finally, if $f, g \in C, f \geqslant g$ and (c) holds, $2 s^{+}=|s|+s$ implies

$$
\begin{aligned}
2 \int_{\Omega}(T(g)-T(f))^{+} & =\int_{\Omega}|T(g)-T(f)|-\int_{\Omega}(T(g)-T(f)) \\
& \leqslant \int_{\Omega}|g-f|-\int_{\Omega}(g-f)=0 .
\end{aligned}
$$


We have proved Lemma 3.2 here for completeness. The parallel result for $L^{\infty}$ and extensions are discussed in [5]. It is recognized that there will be results analogous to those of this paper for, in particular, equations of the form $u_{t}-\Delta \varphi(u)=0$ and $u_{t}+f(\operatorname{grad} u)=0$; and, time permitting, these will be developed.

4. The Entropy Condition. In this section we establish that if $u^{\Delta t}$ is an approximation of a solution of $(0.1)$ produced by a monotone conservation-form difference scheme via the prescription (3.3); and there is a sequence $\Delta t_{l} \rightarrow 0$ for which $u^{\Delta t_{l}}$ converges to a limit $u$ boundedly a.e., then $u$ is an entropy solution of $(0.1)$. In the next section we show that every sequence $\Delta t_{l}$ convergent to zero has a subsequence with the above property, and then deduce that $u^{\Delta t}$ converges as $\Delta t \rightarrow 0$ from the uniqueness Theorem 2.1 .

If $\Delta t_{l}$ is given, it determines the lattices $\{(j \Delta x, k \Delta y)\}=\left\{\left(j / \lambda^{x}, k / \lambda^{y}\right) \Delta t_{l}\right\}$, $\left\{\left(j \Delta x, k \Delta y, n \Delta t_{l}\right)\right\}=\left\{\left(j / \lambda_{x}, k / \lambda^{y}, n\right) \Delta t_{l}\right\}$ and the associated partitions $\left\{R_{j, k}\right\},\left\{R_{j, k}\right.$ $\left.\times\left[n \Delta t_{l},(n+1) \Delta t_{l}\right)\right\}$ of $\mathbf{R}^{2}$ and $\mathbf{R}^{2} \times[0, \infty)$. These depend on $l$, but this will not be explicitly indicated by our notation. The ratios $\lambda^{x}, \lambda^{y}$ will be held constant (so $\Delta x, \Delta y$ depend on $l$ ). As $l$ varies, so too will the initial data involved in computing $u^{\Delta t_{l}}$; but this will not be indicated either.

PROPOSITION 4.1. Let (3.1) be a conservation-form difference approximation consistent with (0.1) which is monotone on $[a, b]$ and has continuous numerical fluxes $g_{1}, g_{2}$. Suppose a sequence $\Delta t_{l}$ of positive numbers convergent to 0 is given. Let $u^{\Delta t_{l}}$ be computed by (3.3), $a \leqslant U_{j, k}^{0} \leqslant b$, and $u^{\Delta t_{l}} \rightarrow u$ boundedly a.e. on $\mathbf{R}^{2} \times$ $[0, T]$. Then, $u$ is an entropy solution of $u_{t}+f_{1}(u)_{x}+f_{2}(u)_{y}=0$ on $\mathbf{R}^{2} \times[0, T]$.

Lax and Wendroff [15] observed that under the above assumptions $u$ will be a weak solution of the equation (even if $G$ is not monotone). We require the following simple lemma which is at the crux of their argument:

LEMMA 4.2. Let $\Delta t_{l} \rightarrow 0$ and

$$
\lim _{l \rightarrow \infty} \sum_{n=0}^{\infty} \sum_{j, k} V_{j, k}^{n} \chi_{j, k}^{n}=v
$$

boundedly a.e. on $\mathbf{R}^{2} \times[0, \infty)$. Let

$$
\varphi \in C_{0}^{1}\left(\mathbf{R}^{2} \times(0, \infty)\right) \text { and } \varphi_{j, k}^{n}=\varphi\left(j \Delta x, k \Delta y, n \Delta t_{l}\right) .
$$

Then

$$
\lim _{l \rightarrow \infty} \sum_{n=0}^{\infty} \sum_{j, k} \varphi_{j, k}^{n}\left(\Delta_{+}^{z} V\right)_{j, k}^{n} \Delta x \Delta y \Delta t_{l}=-\int_{0}^{\infty} \int_{\mathbf{R}^{2}}\left(\frac{\partial \varphi}{\partial z}\right) v d x d y d t
$$

for $z=x, y, t$.

The proof is elementary and is omitted. (While this lemma is not formulated in [15], the arguments there suffice for the proof.)

Proof of Proposition 4.1. Our proof follows the strategy of the one given by Harten, Hyman and Lax [10] for $N=1$. Given $c \in \mathbf{R}$, we will produce continuous numerical entropy fluxes $h_{1}\left(\alpha_{-p,-r}, \ldots, \alpha_{q, s+1}\right), h_{2}\left(\alpha_{-p,-r}, \ldots, \alpha_{q+1, s}\right)$ such that $h_{i}(u, \ldots, u)=\operatorname{sgn}(u-c)\left(f_{i}(u)-f_{i}(c)\right)$ and 


$$
\begin{aligned}
\Delta_{+}^{t}\left|U_{j, k}^{n}-c\right|+\lambda^{x} \Delta_{+}^{x} h_{1}\left(U_{j-p, k-r}^{n}, \ldots, U_{j+q, k+s+1}^{n}\right) \\
+\lambda^{y} \Delta_{+}^{y} h_{2}\left(U_{j-p, k-r}^{n}, \ldots, U_{j+q+1, k+s}^{n}\right) \leqslant 0 .
\end{aligned}
$$

Once this is done, Proposition 4.1 follows. Indeed, multiply (4.2) by $\Delta x \Delta y \Delta t_{l} \varphi_{j, k}^{n} \geqslant$ 0 and sum. Setting, respectively, $V_{j, k}^{n}=\left|U_{j, k}^{n}-c\right|, h_{1}\left(U_{j-p, k-r}^{n}, \ldots, U_{j+q, k+s+1}^{n}\right)$, $h_{2}\left(U_{j-p, k-r}^{n}, . ., U_{j+q+1, k+s}^{n}\right)$ we can take respectively, $v=|u-c|$, $\operatorname{sgn}(u-c)\left(f_{1}(u)-c\right), \operatorname{sgn}(u-c)\left(f_{2}(u)-c\right)$ in Lemma 4.1. Letting $l \rightarrow \infty$ and using Lemma 4.1 yields

$$
\begin{aligned}
-\int_{0}^{\infty} \int_{\mathrm{R}^{2}} \varphi_{t}|u-c|+\varphi_{x} & \operatorname{sgn}(u-c)\left(f_{1}(u)-f_{1}(c)\right) \\
& +\varphi_{y} \operatorname{sgn}(u-c)\left(f_{2}(u)-f_{2}(c)\right) d x d y d t \leqslant 0,
\end{aligned}
$$

which is the entropy condition (2.2).

It remains to produce $h_{1}, h_{2}$. Here we improve on [10] in simplicity and generality. With $z \vee w=\max (z, w), z \wedge w=\min (z, w)$ we set

$$
\left\{\begin{array}{l}
h_{1}\left(U_{j-p, k-r}, \ldots, U_{j+q, k+s+1}\right)=g_{1}\left(c \vee U_{j-p, k-r}, \ldots, c \vee U_{j+q, k+s+1}\right) \\
\quad-g_{1}\left(c \wedge U_{j-p, k-r}, \ldots, c \wedge U_{j+q, k+s+1}\right), \\
h_{2}\left(U_{j-p, k-r}, \ldots, U_{j+q+1, k+s}\right)=g_{2}\left(c \vee U_{j-p, k-r}, \ldots, c \vee U_{j+q+1, k+s}\right) \\
\quad-g_{2}\left(c \wedge U_{j-p, k-r}, \ldots, c \wedge U_{j+q+1, k+s}\right) .
\end{array}\right.
$$

A direct calculation using only the definitions yields the identity

$$
\begin{aligned}
\left|U_{j, k}^{n}-c\right|- & \lambda^{x} \Delta_{+}^{x} h_{1}\left(U_{j-p, k-r}^{n}, \ldots\right)-\lambda^{y} \Delta_{+}^{y} h_{2}\left(U_{j-p, k-r}^{n}, \ldots\right) \\
= & G\left(c \vee U_{j-p, k-r}^{n}, \ldots, c \vee U_{j+q+1, k+s+1}^{n}\right) \\
& -G\left(c \wedge U_{j-p, k-r}^{n}, \ldots, c \wedge U_{j+q+1, k+s+1}^{n}\right) .
\end{aligned}
$$

We also have the relations

$$
\left\{\begin{array}{l}
U_{j, k}^{n+1}=G\left(U_{j-p, k-r}^{n}, \ldots, U_{j+q+1, k+s+1}^{n}\right) \\
c=G(c, c, \ldots, c)
\end{array}\right.
$$

From (4.5) and the monotonicity of $G$ we find

$$
\left\{\begin{array}{l}
c \vee U_{j, k}^{n+1} \leqslant G\left(c \vee U_{j-p, k-r}^{n}, \ldots, c \vee U_{j+q+1, k+s+1}^{n}\right), \\
-\left(c \wedge U_{j, k}^{n+1}\right) \leqslant-G\left(c \wedge U_{j-p, k-r}^{n}, \ldots, c \wedge U_{j+q+1, k+s+1}^{n}\right) .
\end{array}\right.
$$

Adding the inequalities (4.6) and entering the result in (4.4) yields (4.2). This completes the proof. 
5. The Proof of Theorem 1. We will actually prove more than Theorem 1 states here, for we will not need to assume the existence of the solution $S(t) u_{0}$ of $(0.1)$. This will follow from our proofs. To begin, we assume that $a \leqslant u_{0} \leqslant b$ a.e., $u_{0} \in$ $B V\left(\mathbf{R}^{2}\right)$ and $u_{0}$ has compact support. Let $u^{\Delta t}$ be given by (3.3) with $U^{0}=u_{0 \Delta}$; see (2.13).

From the stability estimates of Proposition 3.1 and (2.14)-(2.17) we deduce

$$
\left\{\begin{array}{l}
\left\|u^{\Delta t}(\cdot, \cdot, t)\right\|_{Z} \leqslant\left\|u_{0}\right\|_{Z} \quad \text { for } Z=L^{1}\left(\mathbf{R}^{2}\right), B V\left(\mathbf{R}^{2}\right), \text { and } \\
a \leqslant u^{\Delta t} \leqslant b \text { everywhere. }
\end{array}\right.
$$

Also, because $\lambda^{x}, \lambda^{y}$ are fixed, it is easy to see that there is a $c_{0}>0$ such that if $u_{0}$ vanishes for $|x|+|y| \geqslant R$, then

$$
u^{\Delta t}(x, y, t)=0 \text { for }|x|+|y| \geqslant R+c_{0}+c_{0} t, 0 \leqslant \Delta t \leqslant 1 \text {. }
$$

Because bounded subsets of $B V\left(\mathbf{R}^{2}\right) \cap L^{1}\left(\mathbf{R}^{2}\right)$ are precompact in $L_{\text {loc }}^{1}\left(\mathbf{R}^{2}\right)$ (see (2.9)), (5.1) and (5.2) imply that if $T>0$, then

$$
\left\{u^{\Delta t}(\cdot, \cdot, t): 0 \leqslant t \leqslant T, 0 \leqslant \Delta t \leqslant 1\right\} \text { is precompact in } L^{1}\left(\mathbf{R}^{2}\right) .
$$

Next the estimate of Corollary 3.5 supplies us with

$$
\left\|u^{\Delta t}\left(\cdot, \cdot, t_{1}\right)-u^{\Delta t}\left(\cdot, \cdot, t_{2}\right)\right\|_{L^{1}\left(\mathrm{R}^{2}\right)} \leqslant c\left(\left|t_{1}-t_{2}\right|+2 \Delta t\right)\left\|u_{0}\right\|_{B V\left(\mathrm{R}^{2}\right)} .
$$

By the proof of Arzela-Ascoli's theorem, (5.3) and (5.4) guarantee that if $\Delta t_{l} \rightarrow 0$, then there is a subsequence $\Delta t_{l(k)}$ and a function $u:[0, \infty) \rightarrow L^{1}\left(\mathbf{R}^{2}\right)$ such that

$$
\lim _{k \rightarrow \infty} \max _{0 \leqslant t \leqslant T}\left\|u^{\Delta t_{l}(k)}(\cdot, \cdot, t)-u(\cdot, \cdot, t)\right\|_{L^{1}\left(\mathrm{R}^{2}\right)}=0 \text { for } T>0 .
$$

From (5.1) and (5.4) we deduce $a \leqslant u \leqslant b$ a.e. and

$$
\left\|u\left(\cdot, \cdot, t_{1}\right)-u\left(\cdot, \cdot, t_{2}\right)\right\|_{L^{1}\left(\mathrm{R}^{2}\right)} \leqslant c\left|t_{1}-t_{2}\right|\left\|u_{0}\right\|_{B V\left(\mathrm{R}^{2}\right)} \cdot
$$

Passing to a further subsequence if necessary, we can have $u^{\Delta t_{l}(k)} \rightarrow u$ boundedly a.e. By Proposition 4.1, $u$ is an entropy solution of the conservation law in (0.1). By (5.6), and $u(\cdot, \cdot, 0)=u_{0}, u$ assumes the initial value $u_{0}$ continuously in $L^{1}\left(\mathbf{R}^{2}\right)$. The uniqueness Theorem 2.1 guarantees then the uniqueness of $u$, and we conclude

$$
\lim _{\Delta t \rightarrow 0}\left\|u^{\Delta t}(\cdot, \cdot, t)-u(\cdot, \cdot, t)\right\|_{L^{1}\left(\mathbf{R}^{2}\right)}=0 \text { uniformly for bounded } t .
$$

This completes the analysis when $u_{0}$ has compact support.

For general $u_{0} \in L^{1}\left(\mathbf{R}^{2}\right)$ with $a \leqslant u_{0} \leqslant b$ a.e. we choose $u_{0, m} \in B V\left(\mathbf{R}^{2}\right) \cap$ $L^{1}\left(\mathbf{R}^{2}\right)$ with compact support such that

$$
\left\{\begin{array}{l}
a \leqslant u_{0, m} \leqslant b \text { and } \\
\left\|u_{0}-u_{0, m}\right\|_{L}{ }_{\left(\mathbf{R}^{2}\right)} \rightarrow 0 \text { as } m \rightarrow \infty
\end{array}\right.
$$

This can be done by any standard method. Denote the difference scheme solution corresponding to the initial-value $u_{0, m}$ by $u_{m}^{\Delta t}$. Below, we regard all functions as functions of $t$ with values in $L^{1}\left(\mathbf{R}^{2}\right)$. We know that 


$$
\lim _{\Delta t \rightarrow 0} u_{m}^{\Delta t}(t)=u_{m}(t) \text { exists uniformly for bounded } t
$$

Moreover, from Proposition 3.1(c) it follows that

$$
\sup _{t \geqslant 0}\left\|u_{m}^{\Delta t}(t)-u_{l}^{\Delta t}(t)\right\|_{L^{1}\left(\mathrm{R}^{2}\right)} \leqslant\left\|u_{0, m}-u_{0, l}\right\|_{L^{1}\left(\mathrm{R}^{2}\right)}
$$

and so $\left\{u_{m}\right\}$ is Cauchy in $C\left([0, \infty): L^{1}\left(\mathbf{R}^{2}\right)\right)$ and hence converges uniformly to a limit $u \in C\left([0, \infty): L^{1}\left(\mathbf{R}^{2}\right)\right)$. Finally,

$$
\begin{aligned}
& \left\|u^{\Delta t}(t)-u(t)\right\|_{L^{1}\left(\mathrm{R}^{2}\right)} \\
& \quad \leqslant\left\|u^{\Delta t}(t)-u_{m}^{\Delta t}(t)\right\|_{L^{1}\left(\mathrm{R}^{2}\right)}+\left\|u_{m}^{\Delta t}(t)-u_{m}(t)\right\|_{L^{1}\left(\mathrm{R}^{2}\right)}+\left\|u_{m}(t)-u(t)\right\|_{L^{1}\left(\mathrm{R}^{2}\right)} \\
& \quad \leqslant\left\|u_{0}-u_{0, m^{2}}\right\|_{L^{1}\left(\mathrm{R}^{2}\right)}+\left\|u_{m}^{\Delta t}(t)-u_{m}(t)\right\|_{L^{1}\left(\mathrm{R}^{2}\right)}+\left\|u_{m}(t)-u(t)\right\|_{L^{1}\left(\mathrm{R}^{2}\right)} .
\end{aligned}
$$

The first and third terms above can be made small uniformly in $t$ by taking $m$ large. Then the middle term can be made small by taking $\Delta t$ small (uniformly for $t$ bounded). Hence $\lim _{\Delta t \rightarrow 0} u^{\Delta t}=u$ locally uniformly in $L^{1}\left(\mathbf{R}^{2}\right)$ (and $u$ is continuous into $L^{1}\left(\mathbf{R}^{2}\right.$ ) by its construction). This completes the proof.

A reexamination of the proof of Theorem 5.1 and the various ingredients of the preceding sections shows that we have in fact proved the following results on existence and properties of solutions of (0.1) (given the uniqueness Theorem 2.1).

COROLLARY 5.1. Let the conservation law have Lipschitz continuous fluxes $f_{i}$. Then for every initial-data $u_{0} \in L^{1}\left(\mathbf{R}^{N}\right) \cap L^{\infty}\left(\mathbf{R}^{N}\right)$ there is a unique entropy solution $u \in C\left([0, \infty): L^{1}\left(\mathbf{R}^{N}\right)\right)$ of $(0.1)$ with $u(0)=u_{0}$. Denoting this solution by $S(t) u_{0}$ we have:

(a) If $u_{0} \in B V\left(\mathbf{R}^{N}\right), t \rightarrow S(t) u_{0}$ is Lipschitz continuous into $L^{1}\left(\mathbf{R}^{N}\right)$ and $\left\|S(t) u_{0}\right\|_{B V\left(\mathrm{R}^{N}\right)} \leqslant\left\|u_{0}\right\|_{B V\left(\mathrm{R}^{N}\right)}$.

(b) $\left\|S(t) u_{0}-S(t) v_{0}\right\|_{L^{1}\left(\mathrm{R}^{N}\right)} \leqslant\left\|u_{0}-v_{0}\right\|_{L^{1}\left(\mathrm{R}^{N}\right)}$.

(c) $u_{0} \leqslant v_{0}$ a.e. implies $S(t) u_{0} \leqslant S(t) v_{0}$ a.e.

(d) $a \leqslant u_{0} \leqslant b$ a.e. implies $a \leqslant S(t) u_{0} \leqslant b$ a.e.

All of the above is well known, even in much greater generality; see [13], in particular. Crandall [4] and Benilan [1] also treat cases in which the $f_{i}$ need not be Lipschitz. In fact, it is enough that the $f_{i}$ are continuous and

$$
\lim _{r \rightarrow 0}\left|f_{i}(r)-f_{i}(0)\right| /|r|(N-1) / N=0
$$

in order that Theorem 2.1 hold with $u_{i 0} \in L^{\infty}\left(\mathbf{R}^{N}\right) \cap L^{1}\left(\mathbf{R}^{N}\right)$, provided $R=\infty$ in (2.3) and (2.4). In order to compute solutions of (0.1) in this non-Lipschitz case, one would have to approximate $\left(f_{1}, \ldots, f_{N}\right)$ by smoother functions $\left(f_{1}^{l}, \ldots, f_{N}^{l}\right)$, solve a difference approximation to the resulting problem with $\lambda_{l}^{x}, \lambda_{l}^{y}$ chosen appropriately, and then let $l \rightarrow \infty, \Delta t \rightarrow 0$. The modulus of continuity in time must be treated appropriately, but we will not consider this here. 
6. The Inhomogeneous Equation. We briefly remark on how the analysis given above can be carried out for the more general problem

$$
\left\{\begin{array}{l}
u_{t}+\sum_{i=1}^{N} f_{i}(u)_{x_{i}}=F(x, t), \\
u(x, 0)=u_{0}(x) .
\end{array}\right.
$$

The corresponding difference schemes have the form (for $N=2$ )

$$
U_{j, k}^{n+1}=G\left(U_{j-p, k-r}^{n}, \ldots, U_{j+q+1, k+s+1}^{n}\right)+\Delta t F_{j, k}^{n},
$$

or

$$
U^{n+1}=\vec{G}\left(U^{n}\right)+\Delta t F^{n},
$$

where $G$ satisfies the conditions of the preceding sections.

THEOREM 6.1. Let $G$ define a conservation-form difference scheme consistent with (0.1), which is monotone on $[a, b]$ and has Lipschitz continuous fluxes. Let $T$ $>0, u_{0} \in L^{\infty}\left(\mathbf{R}^{2}\right) \cap L^{1}\left(\mathbf{R}^{2}\right)$ and $F \in L^{\infty}\left(\mathbf{R}^{2} \times(0, T)\right) \cap L^{1}\left(\mathbf{R}^{2} \times(0, T)\right)$ satisfy a $+T\|F\|_{L^{\infty}} \leqslant u_{0} \leqslant b-\|F\|_{L^{\infty}} T$ a.e. Let $U^{0}=u_{0 \Delta}$ and

$$
F_{j, k}^{n}=\frac{1}{\Delta t \Delta x \Delta y} \int_{n \Delta t}^{(n+1) \Delta t} \int_{R_{j, k}} F(x, y, t) d x d y d t .
$$

Then $U^{n}$, defined by (6.3) satisfies $a \leqslant U_{j, k}^{n} \leqslant b$ for all $n, j, k$ such that $n \Delta t \leqslant T$ and

$$
u^{\Delta t}=\sum_{n=0}^{\infty} \sum_{j, k} U_{j, k}^{n} \chi_{j, k}^{n}
$$

converges in $L^{1}\left(\mathbf{R}^{2}\right)$ uniformly on compact subsets of $[0, T)$ to the unique entropy solution of (6.1).

Sketch of Proof. In addition to the arguments in preceding sections we require a uniqueness theorem (see [13] for this) and a few estimates given now. From Proposition 3.1 we deduce immediately

$$
\left\{\begin{array}{l}
\sup _{j, k} U_{j, k}^{n+1} \leqslant \sup _{j, k} U_{j, k}^{n}+\Delta t \sup _{j, k} F_{j, k}^{n}, \\
\inf _{j, k} U_{j, k}^{n+1} \geqslant \inf _{j, k} U_{j, k}^{n}+\Delta t \inf _{j, k} F_{j, k}^{n}
\end{array}\right.
$$

and

$$
\left\|U^{n+1}\right\|_{X} \leqslant\left\|U^{n}\right\|_{X}+\Delta t\left\|F^{n}\right\|_{X}
$$

for $X=L^{1}(\Delta)$ and $X=B V(\Delta)$. Moreover, if $\hat{U}^{n+1}=G\left(\hat{U}^{n}\right)+\Delta t \hat{F}^{n}$, we find

$$
\left\|U^{n}-\hat{U}^{n}\right\|_{L^{1}(\Delta)} \leqslant\left\|U^{0}-\hat{U}^{0}\right\|_{L^{1}(\Delta)}+\sum_{j=0}^{n-1} \Delta t\left\|F^{j}-\hat{F}^{j}\right\|_{L^{1}(\Delta)} .
$$

Finally, the estimate of Proposition 3.4 is replaced (with $n \Delta t \leqslant t_{2}<(n+1) \Delta t, m \Delta t$ $\leqslant t_{1}<(m+1) \Delta t$ and $m \leqslant n$ as before) by 


$$
\begin{aligned}
\int_{\mathrm{R}^{2}}\left|u^{\Delta t}\left(x, y, t_{2}\right)-u^{\Delta t}\left(x, y, t_{1}\right)\right| d t \\
=\left\|U^{n}-U^{m}\right\|_{L^{1}(\Delta)} \leqslant \sum_{j=m}^{n-1}\left\|U^{j+1}-U^{j}\right\|_{L^{1}(\Delta)} \\
=\sum_{j=m}^{n-1}\left\|\vec{G}\left(U^{j}\right)+\Delta t F^{j}-U^{j}\right\|_{L^{1}(\Delta)} \\
\leqslant \sum_{j=m}^{n-1}\left\|\vec{G}\left(U^{j}\right)-U^{j}\right\|_{L^{1}(\Delta)}+\sum_{j=m}^{n-1} \Delta t\left\|F_{j}\right\|_{L^{1}(\Delta)}
\end{aligned}
$$

Now we use Proposition 3.5 and (6.5) to estimate the right-hand side above by

$$
\Delta t(n-m) \text { cons. }\left(\left\|U^{0}\right\|_{B V(\Delta)}+\sum_{j=0}^{n-1} \Delta t\left\|F^{j}\right\|_{B V(\Delta)}\right)+\sum_{j=m}^{n-1} \Delta t\left\|F_{j}\right\|_{L^{1}(\Delta)} .
$$

Thus, if

$$
\int_{0}^{T}\|F(\cdot, \cdot, t)\|_{B V\left(\mathbf{R}^{2}\right)} d t<\infty,
$$

we have the type of equicontinuity of $u^{\Delta t}$ as $\Delta t \rightarrow 0$ used in the proof of Theorem 1 ; and the proof may be completed much as before. (One begins, say, with $u_{0} \in$ $C_{0}^{\infty}\left(\mathbf{R}^{2}\right), F \in C_{0}^{\infty}\left(\mathbf{R}^{2} \times[0, T)\right)$ and passes to the general case via (6.6).)

\section{Mathematics Research Center University of Wisconsin, Madison Madison, Wisconsin 53706}

Department of Mathematics University of California, Berkeley Berkeley, California 94720

1. PH. BENILAN, Equation d'Evolution dans un Espace de Banach Quelconque, Thesis, Université de Orsay, 1972.

2. S. BURSTEIN, P. D. LAX \& G. SOD, Lectures on Combustion Theory, Courant Mathematics and Computing Laboratory Report, September 1978.

3. E. CONWAY \& J. SMOLLER, "Global solutions of the Cauchy problem for quasilinear first order equations in several space variables," Comm. Pure Appl. Math., v. 19, 1966, pp. 95-105.

4. M. G. CRANDALL, "The semigroup approach to first order quasilinear equations in several space variables," Israel J. Math., v. 12, 1972, pp. 108-132.

5. M. G. CRANDALL \& L. TARTAR, "Some relations between non expansive and order preserving mappings." (To appear.)

6. A. DOUGLIS, Lectures on Discontinuous Solutions of First Order Nonlinear Partial Differential Equations in Several Space Variables, North British Symposium on Partial Differential Equations, 1972.

7. N. DUNFORD \& J. T. SCHWARTZ, Linear Operators, Part 1 : General Theory, Pure and Appl. Math., Vol. 7, Interscience, New York, London, 1958.

8. S. K. GODUNOV, "Finite difference methods for numerical computations of discontinuous solution of equations of fluid dynamics," Mat. Sb., v. 47, 1959, pp. 271-295. (Russian)

9. A. HARTEN, "The artificial compression method for computation of shocks and contact discontinuities: I. Single conservation laws," Comm. Pure Appl. Math., v. 39, 1977, pp. 611-638.

10. A. HARTEN, J. M. HYMAN \& P. D. LAX, "On finite difference approximations and entropy conditions for shocks," Comm. Pure Appl. Math., v. 29, 1976, pp. 297-322. 
11. G. JENNINGS, "Discrete shocks," Comm. Pure Appl. Math., v. 27, 1974, pp. 25-37.

12. K. KOJIMA, "On the existence of discontinuous solutions of the Cauchy problem for quasilinear first order equations," Proc. Japan Acad., v. 42, 1966, pp. 705-709.

13. S. N. KRUŽKOV, "First order quasilinear equations with several space variables," Math. USSR Sb., v. 10, 1970, pp. 217-243.

14. P. D. LAX, Hyperbolic Systems of Conservation Laws and the Mathematical Theory of Shock Waves, SIAM Regional Conference Series in Applied Mathematics \#11.

15. P. D. LAX \& B. WENDROFF, "Systems of conservation laws," Comm. Pure Appl. Math., v. 13, 1960, pp. 217-237.

16. A. Y. LE ROUX, "A numerical conception of entropy for quasi-linear equations," Math. Comp., v. 31, 1977, pp. 848-872.

17. A. MAJDA \& M. CRANDALL, Numer. Math. (To appear.)

18. A. MAJDA \& S. OSHER, "Numerical viscosity and the entropy condition," Comm. Pure Appl. Math. (To appear.)

19. S. OHARU \& T. TAKAHASHI, "A convergence theorem of nonlinear semigroups and its application to first order quasilinear equations," J. Math. Soc. Japan, v. 26, 1974, pp. 124-160.

20. O. A. OLEINIK, "Discontinuous solutions of nonlinear differential equations," Amer. Math. Soc. Transl. (2), v. 26, 1963, pp. 95-172.

21. G. STRANG, "On the construction and comparison of difference schemes," SIAM J. Numer. Anal., v. 15, 1968, pp. 506-517.

22. A. I. VOL'PERT, "The spaces $B V$ and quasilinear equations," Math. USSR Sb., v. 2, 1967, pp. 225-267.

23. N. N. KUZNECOV \& S. A. VOLOŠIN, “On monotone difference approximations for a first-order quasi-linear equation,” Soviet Math. Dokl., v. 17, 1976, pp. 1203-1206. 